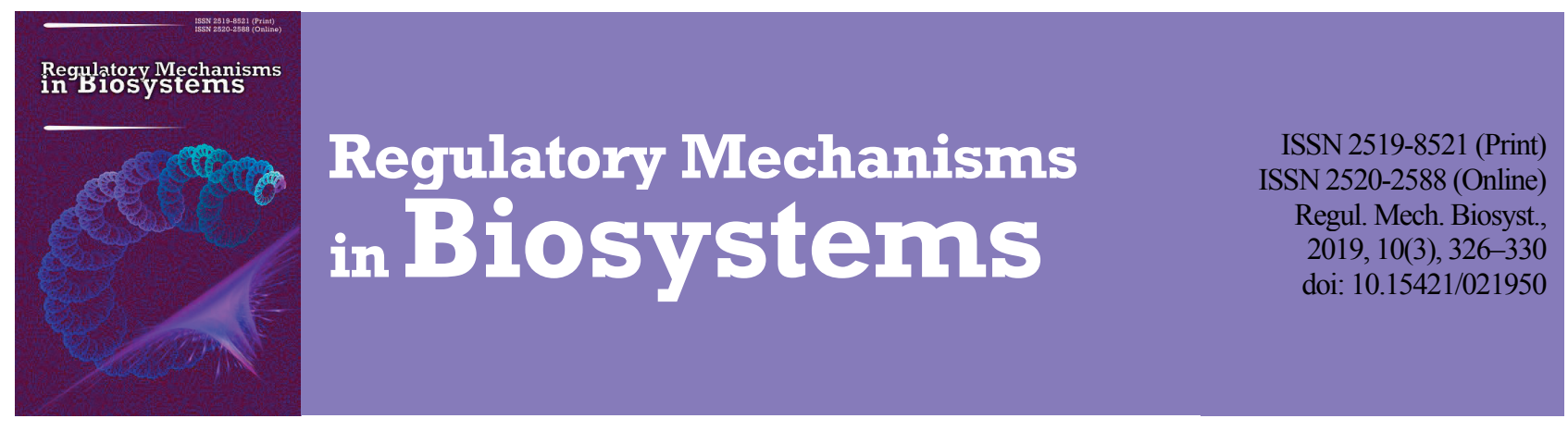

\title{
Evaluation of the effectiveness of cryptic coloration of the Carolina anole's skin
}

\author{
A. B. Kiladze \\ A. N. Severtsov Institute of Ecology and Evolution, Russian Academy of Sciences, Moscow, Russia
}

Article info

Received 17.07.2019

Received in revised form 20.08.2019

Accepted 21.08.2019

\author{
Kiladze, A. B. (2019). Evaluation of the effectiveness of cryptic coloration of the Carolina anole's skin. Regulatory Mechanisms in \\ Biosystems, 10(3), 326-330. doi:10.15421/021950
}

Cryptic coloration of animals' integument is one of the effective adaptations that allow them to lead an active lifestyle while being protected from natural enemies due to visual disguise. This is achieved by the similarity of body color of a particular individual to the background of various substrates in its environment. The morphological and functional basis of cryptic coloration in vertebrates, including reptiles, is ensured by the skin pigmentation. Using bioinformatic methods, we calculated the skin camouflage index of the Carolina

A. N. Severtsov Institute of Ecology and Evolution Russian Academy of Sciences, Moscow, 119071, Russia.

Tel.: +7-916-503-44-51. E-mail:andreykiladze@ yandex.ru anole (Anolis carolinensis Voigt, 1832) in various conditions of its habitat. The skin camouflage index (Ic) is the ratio of the sum of the average values of rgb coordinates of the skin color to the sum of the average values of rgb coordinates of the color of the external substrate. Ic satisfies the effective level of adaptation to habitat conditions if it falls within the range of 0.80-1.20. It has been shown that rgbvalues of the dominant color of the dorsal skin of green anoles slightly differ from the similar parameters characteristic of the deciduous habitat, which is reflected by Ic of its skin (0.94). In the brown anoles on a background of woody substrate, the Ic value of its skin (0.88) is also optimal, since it exceeds the lower limit (0.80), which indicates close values of the sums of the rgb coordinates of the skin color and the color of the external background. In the mixed green-brown anoles, the deciduous habitat is preferable to the woody one. In the first case, Ic (1.11) is in the optimum zone, and in the second case, Ic (0.70) goes beyond the lower limit of the optimum, which indicates a greater vulnerability of the animal to external threats. We have confirmed the relativity of visual hiding of the skin that is effective only in the habitat conditions in which the camouflage abilities of the skin manifest as fully as possible.

Keywords: adaptations; skin camouflage index; pigmentation; protective coloration; computational biology.

\section{Introduction}

Adaptation of animals is an important factor in the success of life strategies in specific habitat conditions, which, according to the Charles Darwin's (1809-1882) theory, is the result of evolutionary transformations (Gardner, 2009). Biological adaptations are relative in their nature, which determines the preservation of life only for those animals that are the most adapted to a particular system of environmental factors (Ghiselin, 1966; Vitt, 1981). Obviously, in other living conditions, these adaptations would not have the necessary practical value (Ghiselin, 1966).

Cryptic coloration, as an example of effective adaptation to a particular habitat, allows many animals, on the one hand, to ensure opportunities for hunting potential prey, and, on the other hand, to protect themselves from predators (Stevens \& Merilaita, 2009, 2011). In both cases, masking the skin according to the environment, which is an example of passive protection, is the necessary condition for successful vital activity (Norris, 1967; Talbot, 1977; Henry et al., 2008). The issue of cryptic coloration in animals has been reviewed the most completely in the fundamental monograph by Hugh Cott (1900-1987) "Adaptive coloration in animals" (Cott, 1940).

Actual issues of evolution of color polymorphism of the skin, morphological and functional fundamentals, and physiological processes that ensure cryptic coloration of vertebrates are the subject of research both in the field of evolutionary biology (Needham, 1974; Merilaita, 2003; Bond, 2007; Mäthger et al., 2009) and in individual zoological studies (Bagnara et al., 1968; Morrison et al., 1996; Saenko et al., 2013; Allen et al., 2015; Teyssier et al., 2015), to which our study belongs.

The morphological and physiological foundations providing the affinity of the skin color with the habitat lie in the structure and functioning of the skin pigmentation and its derivatives (scales, feathers, hair) (Schneider et al., 2009; Benton et al., 2019). Pigmentation processes that form a specific colored pattern on the surface of the skin are subject to chemical and physical laws of morphogenesis (Turing, 1952; Gierer \& Meinhardt, 1972; Belintsev, 1991). Skin coloration of vertebrates can have signs of symmetry and asymmetry (Chernova \& Kiladze, 2014; Kiladze \& Chernova, 2014), as well as being determined by heterochrony (Chernova \& Kiladze, 2019).

Reptiles, the skin of which in most cases is covered with corneous scales or scutes (Di-Poï \& Milinkovitch, 2016), have acquired well-developed camouflage abilities (Heatwole, 1968; Macedonia et al., 2000) accompanied by a change in color and transformation of the skin pattern (Stuart-Fox \& Moussalli, 2009), which is achieved through a perfect pigmentation system (Bagnara \& Matsumoto, 2006).

Species capable of skin color transformation from bright green to dark brown include the Carolina anole (hereinafter, the anole) (Anolis carolinensis Voigt, 1832). For the anole, stimuli to changing the skin color include not only a change in the amount of light and background color, which is a manifestation of camouflage, but also the nature of activity, stress level, behavioral characteristics, as well as thermoregulation (Hadley \& Goldman, 1969; Claussen \& Art, 1981; Sigmund, 1983; Losos, 2009). The scaly skin of anoles is unique because it demonstrates an example of random keratinization (squamation), while the location of corneous ellipsoid scales is not completely random, since their distribution relative to each other resembles that of the "blue noise" (Landreneau, 2011).

Cryptic coloration is largely determined by the habitat and biotope of the anole that has populated the subtropical southeastern parts of North America including the Atlantic Coastal Plain (North Carolina, South Carolina, Georgia and Florida), as well as the Gulf of Mexico (Alabama, Mississippi, Louisiana and Texas) (Losos, 2009). Typical habitats of the anole include humid forests and glades with shrubs. This anole is mainly a woodland species, but it can also be found on the 
ground. In addition, it can be seen in various anthropogenic landscapes with abundant sunlight, and its color has an affinity for copious foliage, shrubs, and vines (Graeter, 2008). Since anoles tolerate captivity well, they are common objects of commercial demonstration in terrariums at zoological museums, aquariums, and zoos (Murphy, 2017). In addition, they serve as a model species for general biological studies, such as elucidating the physiological foundations of color change (Hadley \& Goldman, 1969) and the morphology of chromatophores (Taylor \& Hadley, 1970) in reptiles.

The aim of our study is to develop a method for quantitative assessment of effective color camouflage of the anole relative to habitat conditions, and it is proposed to use bioinformatic methods based on image analysis, as well as quantitative biology which quantifies many physiological processes.

\section{Materials and methods}

We have studied seven adult animals of the Carolina anole Anolis carolinensis Voigt, 1832 (Squamata: Dactyloidae): animals of both sexes of three color forms kept in the scientific terrarium of the Zoological Museum of Lomonosov Moscow State University (Moscow, Russia) and in the terrarium of the Oceanarium of Antalya (Turkey). We took photographs of each of the lizards having a varying color scheme (from green to brown shades). Fragments of photographs of the skin and of the external habitat (deciduous, deciduous-woody and woody) were processed using software that allows one to define a palette of primary colors, as well as presenting them in rgb coordinates. The software is freely available at www.imgonline.com.ua/get-dominant-colors.php.

The obtained rgb coordinates, which can vary from 0 to 255 (Jones \& Rehg, 2002), were averaged, and the dominant colors characteristic both of anoles of three color forms and of the corresponding habitat were found at www.yandex.ru.

To evaluate the effectiveness of masking in Python 3.7.4 (Farrell, 2019), a code containing the calculation of the skin camouflage index and its limits corresponding to the optimal level of skin color adaptation to the environment was written for one of the averaged rgb coordinates sets characteristic of the green anole and deciduous habitat.

At the first stage, we studied the appearance of the anole under terrarium conditions during its transition from the deciduous crown to the trunk, which was accompanied by a change in the color of the dorsal region of the body from green to brown. At the same time, the tone of coloration fully corresponds to the color of the external substrate where a certain animal is currently located. Of the specific exceptions in color that do not correspond to the background of the habitat, one can name the eye rim, which has an intensely emerald hue, a lighter ventral region, as well as signs of sexual dimorphism manifested in the presence of a white stripe in females. It is necessary to emphasize the relativity of color adaptation, which, on the one hand, provides the necessary level of camouflage, and, on the other hand, distinguishes the animal against a contrasting background of the habitat (Fig. 1), thereby making it not only more noticeable, but also more vulnerable to natural enemies.

Traditionally, when speaking of cryptic coloration, only the fact of the camouflage effect is mentioned, but it is also very important to determine the level of masking efficiency, which will correspond to the degree of adaptation of an animal's skin pigmentation to certain living conditions. To solve this problem, it is first necessary to determine the skin color variability of each form of the anole, and to determine the color variability of the habitat conditions. After processing the fragments of photographs of the dorsal skin and fragments of photographs of the habitat of the lizards we obtained a palette of five colors with their rgb coordinates (Fig. 2).

The palettes we presented are almost identical: the skin colors of the anoles and the external substrate are about the same. This is especially noticeable in green (Fig. 2a) and brown (Fig. 2c) lizards. Differences in the skin and the habitat color for these two forms can relate only to the intensity of the green or brown shades. The borderline form (Fig. 2b) demonstrates polychromatic color, as one part of the palette is close to the color of the deciduous habitat, and the other part - to the woody one. This animal has a mixed color since the main background is green, but with a characteristic brown shadowing effect.
At the second stage, for each case, we averaged individual coordinates for the five colors and obtained the dominant colors of the dorsal region for different forms of anoles, as well as the corresponding colors of their habitat, as well (Table 1).

Based on the new rgb coordinates characterizing the dominant colors, an algorithm for assessing skin camouflage was developed, and the procedure is presented in the form of the following simple code given as an example for the green anole and deciduous habitat, that is:

def $\operatorname{sum}(\mathrm{r} 1, \mathrm{~g} 1, \mathrm{~b} 1)$ :

return $\mathrm{r} 1+\mathrm{g} 1+\mathrm{b} 1$

skin $=\operatorname{sum}(146,231,57)$

print(skin)

def sum (r2, g2, b2):

return $\mathrm{r} 2+\mathrm{g} 2+\mathrm{b} 2$

habitat $=\operatorname{sum}(135,250,79)$

print(habitat)

res $=$ skin $/$ habitat

print(res)

if res $>=0.80$ and $\mathrm{res}<=1.20$ :

print("Optimal level of adaptation to habitat")

else:

print("Non-optimal level of adaptation to habitat")

This program is equivalent to the following formula by which it is possible to calculate the skin camouflage index (Ic), that is, $\mathrm{Ic}=(\mathrm{rl}+\mathrm{gl}+$ b1) / ( $2+\mathrm{g} 2+\mathrm{b} 2)$, where $\mathrm{r} 1, \mathrm{~g} 1, \mathrm{~b} 1$ are the average color coordinates of the skin; r2, g2, b2 - average color coordinates of the substrate (Table 1).

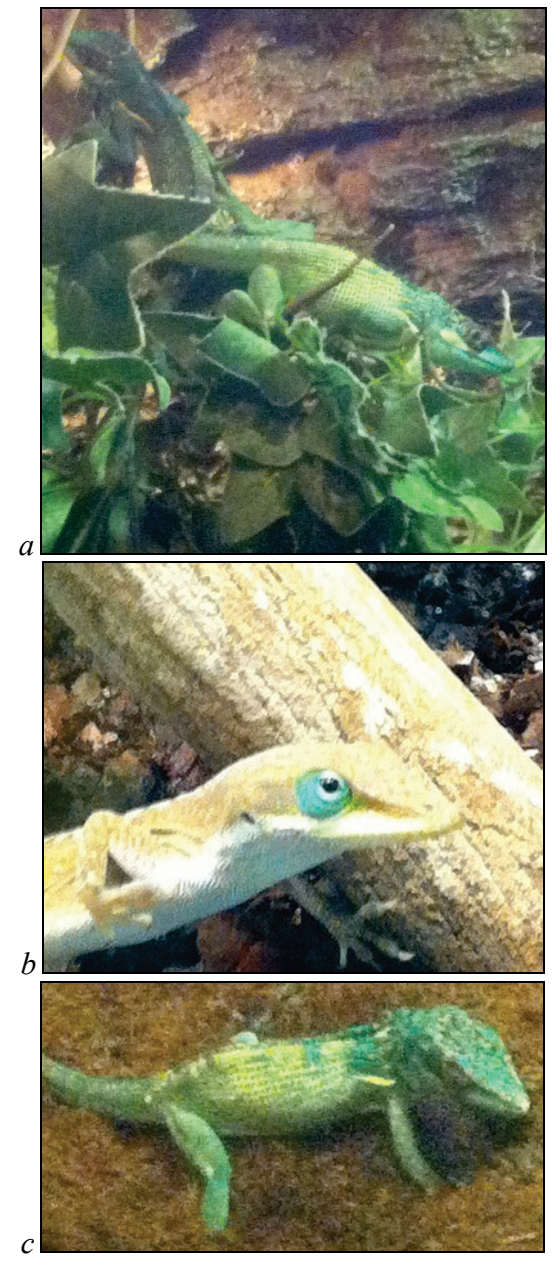

Fig. 1. Green and brown forms of $A$. carolinensis demonstrating the relative nature of skin color adaptation with respect to the external substrate: in the first two cases $(a, b)$, effective camouflage is seen, and in the third $(c)$ one there is no protective coloration; $a, c$-terrarium of the Antalya Oceanarium (Turkey); $b$ - scientific terrarium of the Zoological Museum of Lomonosov Moscow State University (Moscow); photos by A. B. Kiladze 


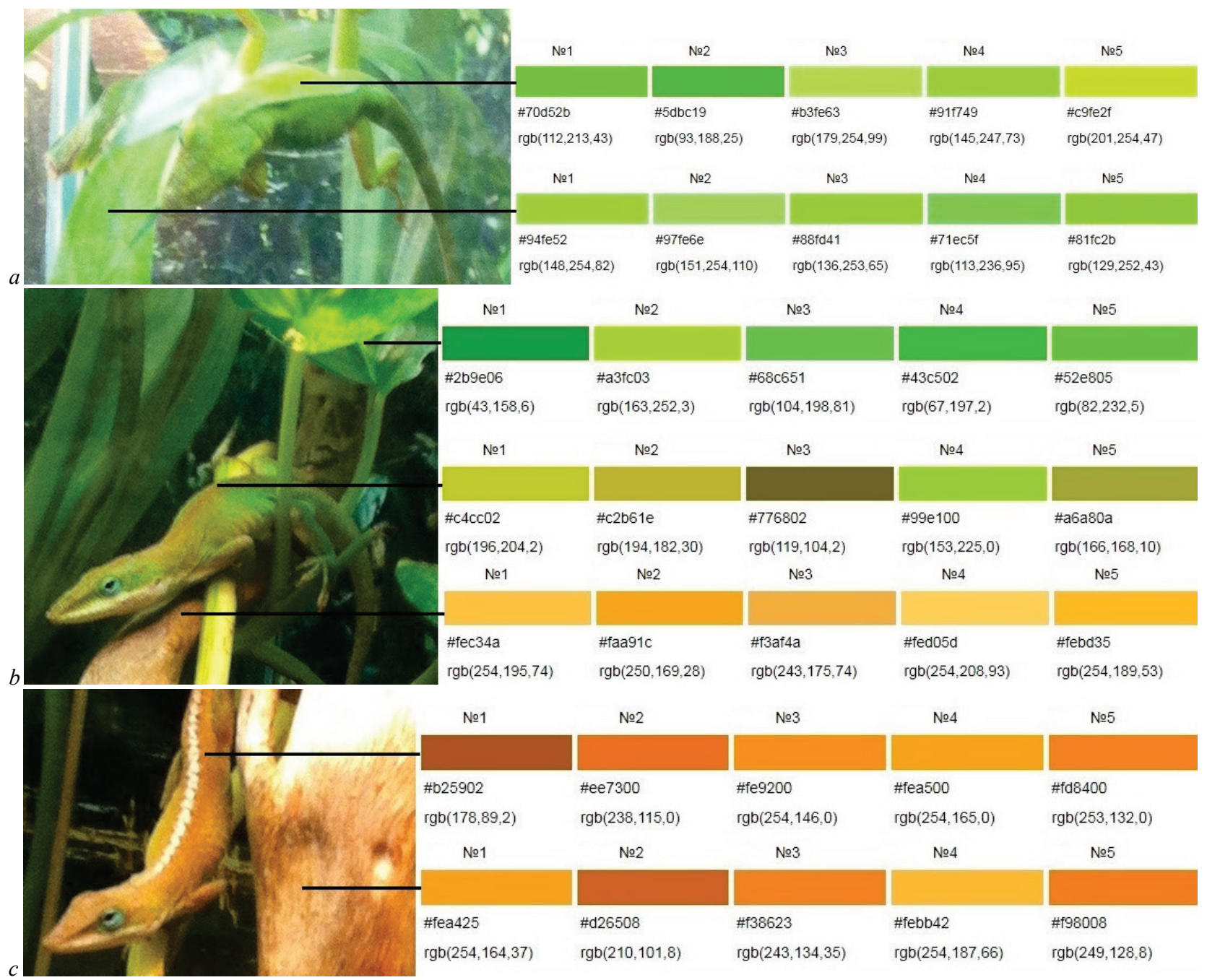

Fig. 2. Green $(a)$, mixed $(b)$ and brown $(c)$ forms of A. carolinensis in various backgrounds of the habitat: on the left: the appearance of lizards from the scientific terrarium of the Zoological Museum of Lomonosov Moscow State University; photos by A. B. Kiladze; on the right: palettes of five colors with their rgb coordinates obtained using the site www.imgonline.com.ua/get-dominant-colors.php

This algorithm is also valid for other color forms of the anole and their corresponding external substrates. We determined the optimality limits of this index as $0.80-1.20$, which indicates an acceptable level of cryptic coloration, as well as acceptable deviations of skin color from the background color of the habitat.

\section{Results}

The pivotal result of this study is to obtain averaged rgb coordinates, on the basis of which we determined not only the dominant colors of the Carolina anole's skin and external habitat, but also calculated the skin camouflage index (Table 1).

\section{Table 1}

Parameters of rgb coordinates for the dominant colors of the dorsal skin of $A$. carolinensis and the background of the habitat

\begin{tabular}{lccc}
\hline \multicolumn{1}{c}{$\begin{array}{c}\text { Color forms } \\
\text { of the species }\end{array}$} & $\begin{array}{c}\text { Parameters of rgb } \\
\text { coordinates for the } \\
\text { dominant color of } \\
\text { the dorsal skin }\end{array}$ & $\begin{array}{c}\text { Parameters of rgb coordi- } \\
\text { nates for the dominant color } \\
\text { of the background of the } \\
\text { habitat }\end{array}$ & $\begin{array}{c}\text { The skin } \\
\text { camouflage } \\
\text { index }\end{array}$ \\
\hline $\begin{array}{l}\text { Green form in } \\
\text { deciduous habitat }\end{array}$ & $\operatorname{rgb}(146,231,57)$ & $\operatorname{rgb}(135,250,79)$ & 0.94 \\
$\begin{array}{l}\text { Mixed form in } \\
\text { deciduous habitat }\end{array}$ & $\operatorname{rgb}(166,177,9)$ & $\operatorname{rgb}(92,207,19)$ & 1.11 \\
$\begin{array}{l}\text { Mixed form in } \\
\text { woody habitat }\end{array}$ & $\operatorname{rgb}(166,177,9)$ & $\operatorname{rgb}(251,187,64)$ & 0.70 \\
$\begin{array}{l}\text { Brown form in } \\
\text { woody habitat }\end{array}$ & $\operatorname{rgb}(235,129,0)$ & $\operatorname{rgb}(242,143,31)$ & 0.88 \\
\hline
\end{tabular}

Analysis of table data showed the following trends: (i) the most adapted anole forms are the green and the brown ones, which is shown by the obtained camouflage indices falling within the range of $0.80-1.20$, while a higher index was obtained for the first form (Ic = 0.94 ) compared to the second one ( $\mathrm{Ic}=0.88$ ); (ii) for the mixed form with a transitional color, deciduous habitat is more preferable than a woody one, that is evidenced by the obtained values of the skin camouflage index. If in first case we can say that there is an optimal level of skin adaptation $(\mathrm{Ic}=1.11)$, then in second case, the index goes beyond the acceptable values $(\mathrm{Ic}=0.70)$ indicating an insufficient affinity of the skin color shades and the habitat being compared.

\section{Discussion}

We believe that the described approaches to assessing the effectiveness of skin camouflage will be in demand in field studies for environmental monitoring of the species because the method is rapid and does not require expensive equipment. The skin camouflage index itself is dimensionless. The above algorithm specifies the index limits, within which we can talk about the optimal level of skin color adaptation to the habitat conditions. In our case, the limits are taken at the level of $20 \%$ variability $(0.80-1.20)$ of the conventional unit. The unit value indicates the maximum coincidence of color shades of the skin and the substrate. If the obtained index goes beyond the given limits, then we can talk about the nonoptimal level of color adaptation of the organism to the habitat conditions. In addition, it is obvious that the occurrence of complete coincidence of colors, when the skin camouflage index is 1 , is not common, therefore, it 
can be considered as quite rare within the population. As part of the discussion, we should also mention certain limitations associated with the calculation of the skin camouflage index. It is important to calculate the sums of the three coordinates and to perform further comparison for similar color shades of the skin and the substrate with a clear camouflaging nature. Obviously, the calculation for definitely different colors that create contrast does not have any biological meaning, while the sum of their rgb coordinates may coincide. For example, compare colors such as $\operatorname{rgb}(150,120,10)$ and $\operatorname{rgb}(120,10,150)$. Their sums coincide, the camouflage index equals 1 ; however, these colors are completely different from each other, therefore, they have no protective ability.

Earlier, we performed a similar study on the cryptic color of the Khorasan agama (redbelly rock agama ) Paralaudakia erythrogastra $(\operatorname{rgb}(177,136,100))$, which is close to the background of the mountain substrate $(\operatorname{rgb}(170,139,128))$, but at that time we did not calculate the skin camouflage index (Kiladze \& Chernova, 2018). While applying the method for calculating the skin camouflage index for the Khorasan agama, one can note a very high level of color approximation to each other, since the index value was Ic $=0.95$, while the obtained value meets the criterion of the optimal color adaptation of the agama skin to the habitat conditions. The color of the agama itself differs from that of the anole in terms of a larger number of spots and stripes, which allows one to speak of a more disruptive coloration (Cott, 1940; Endler, 2006; Amdekar \& Thaker, 2019); however, in the mountain substrate system this form of camouflage is apparently optimal. The anole's color is more uniform and monochrome, which is associated with its natural habitat, as well as with the corneous scales pattern on the surface of its skin.

Concluding the discussion, it can be noted that the problem of statistical and visual modeling, automation and algorithmic presentation for determining the skin colors of animals and humans is a promising area, which is confirmed by long-term studies (Yang et al., 1997; Stevens et al., 2007; Allen et al., 2015; Sanchez et al., 2018; Beltrán, 2019).

\section{Conclusion}

Thus, the proposed method for calculating the skin camouflage index allows us to determine the optimality criteria for the color differences of the Carolina anole's skin with a specific substrate of the habitat, as well as to evaluate color adaptation, which is based on biologically significant protective color determining the success of the life strategy of a species. We believe that this method will find its application in other vertebrates and invertebrates that have the ability for similar morphological and physiological adaptations.

The author is deeply grateful to Dr. O. F. Chernova (Severtsov Institute of Ecology and Evolution, Russian Academy of Sciences) for scientific editing of the manuscript.

\section{References}

Allen, J. J., Akkaynak, D., Sugden, A. U., \& Hanlon, R. T. (2015). Adaptive body patterning, three-dimensional skin morphology and camouflage measures of the slender filefish Monacanthus tuckeri on a Caribbean coral reef. Biological Journal of the Linnean Society, 116(2), 377-396.

Amdekar, M. S., \& Thaker, M. (2019). Risk of social colours in an agamid lizard: Implications for the evolution of dynamic signals. Biology Letters, 15(5), 20190207.

Bagnara, J. T., Taylor, J. D., \& Hadley, M. E. (1968). The dermal chromatophore unit. The Journal of Cell Biology, 38(1), 67-79.

Bagnara, J. T., \& Matsumoto, J. (2006). Comparative anatomy and physiology of pigment cells in nonmammalian tissues. In: Nordlund, J. J., Boissy, R. E., Hearing, V. J., King, R. A., Oetting, W. S., \& Ortonne J.-P. (Eds.). The pigmentary system: Physiology and pathophysiology. Second edition. Blackwell Publishing Ltd, Oxford. Pp. 11-59.

Belintsev, B. N. (1991). Fizicheskie osnovy biologicheskogo formoobfazovaniya [Physical foundation of biological morphogenesis]. Nauka, Moscow (in Russian).

Beltrán, I. (2019). Diurnal colour change in a sexually dimorphic trait in the Andean lizard Anolis heterodermus (Squamata: Dactyloidae). Journal of Natural History, 53, 45-55.

Benton, M. J., Dhouailly, D., Jiang, B., \& McNamara, M. (2019). The early origin of feathers. Trends in Ecology and Evolution, 34(9), 856-869.
Bond, A. B. (2007). The evolution of color polymorphism: Crypticity, searching images, and apostatic selection. Annual Review of Ecology, Evolution, and Systematics, 38, 489-514.

Chernova, O. F., \& Kiladze, A. B. (2014). Symmetry in topography and microstructure of vertebrate skin derivatives. Paleontological Journal, 48, 1284-1294.

Chernova, O. F., \& Kiladze, A. B. (2019). Heterochrony as the basis for inter- and intraspecific diversity of skin in vertebrates. Biology Bulletin Reviews, 9(2), 174-189.

Claussen, D. L., \& Art, G. R. (1981). Heating and cooling rates in Anolis carolinensis and comparisons with other lizards. Comparative Biochemistry and Physiology, Part A: Physiology, 69(1), 23-29.

Cott, H. B. (1940). Adaptive coloration in animals. Methuen \& Co. Ltd., London.

Di-Poï, N., \& Milinkovitch, M. C. (2016). The anatomical placode in reptile scale morphogenesis indicates shared ancestry among skin appendages in amniotes. Science Advances, 2, e1600708.

Endler, J. A. (2006). Disruptive and cryptic coloration. Proceedings of the Royal Society B: Biological Sciences, 273(1600), 2425-2426.

Farrell, P. (2019). Math adventures with Python: An illustrated guide to exploring math with code. No Starch Press, San Francisco.

Gardner, A. (2009). Adaptation as organism design. Biology Letters, 5, 861-864.

Ghiselin, M. T. (1966). On semantic pitfalls of biological adaptation. Philosophy of Science, 33, 147-153.

Gierer, A., \& Meinhardt, H. (1972). A theory of biological pattern formation. Kybernetik, 12(1), 30-39.

Graeter, G. J. (2008). Green anole. In: Jensen, J. B. (Ed.). Amphibians and reptiles of Georgia. University of Georgia Press, Athens. Pp. 296-298.

Hadley, M. E., \& Goldman, J. M. (1969). Physiological color changes in reptiles. The American Zoologist, 9, 489-504.

Heatwole, H. (1968). Relationship of escape behavior and camouflage in anoline lizards. Copeia, 1968(1), 109-113.

Henry, E., Brewer, J., Mougey, K., \& Perry, G. (2008). The Texas horned lizard in Central and Western Texas. Iguana, 15(4), 205-211.

Jones, M. J., \& Rehg, J. M. (2002). Statistical color models with application to skin detection. International Journal of Computer Vision, 46(1), 81-96.

Kiladze, A. B., \& Chernova, O. F. (2014). Symmetry and asymmetry of configuration and structure of vertebrate skin. Paleontological Journal, 48, 1295-1302.

Kiladze, A. B., \& Chernova, O. F. (2018). Adaptive peculiarities of pigmentation in the redbelly rock agama Paralaudakia erythrogastra (Nikolsky, 1896) (Agamidae). Ukrainian Journal of Ecology, 8(4), 248-251.

Landreneau, E. B. (2011). Scales and scale-like structures. Dissertation. Texas A\&M University, College Station. Pp. 32-33.

Losos, J. B. (2009). Lizards in an evolutionary tree: Ecology and adaptive radiation of anoles. University of California Press, Berkeley.

Macedonia, J. M., James, S., Wittle, L. W., \& Clark, D. L. (2000). Skin pigments and coloration in the Jamaican radiation of Anolis lizards. Journal of Herpetology, 34(1), 99-109.

Mäthger, L. M., Denton, E. J., Marshall, N. J., \& Hanlon, R. T. (2009). Mechanisms and behavioral functions of structural coloration in cephalopods. Journal of the Royal Society Interface, 6(Suppl. 2), S149-S163.

Merilaita, S. (2003). Visual background complexity facilitates the evolution of camouflage. Evolution, 57(6), 1248-1254.

Morrison, R. L., Sherbrooke, W. C., \& Frost-Mason, S. K. (1996). Temperaturesensitive, physiologically active iridophores in the lizard Urosaurus ornatus: An ultrastructural analysis of color change. Copeia, 1996(4), 804-812.

Murphy, J. B. (2017). Cuban herps. Herpetological Review, 48(3), 582-588.

Needham, A. E. (1974). The functions of integumental zoochromes. In: The significance of zoochromes. Zoophysiology and Ecology, 3. Springer, Berlin, Heidelberg. Pp. 131-161.

Norris, K. S. (1967). Color adaptation in desert reptiles and its thermal relationships. In: Milstead, W. W. (Ed.). Lizard ecology: A symposium. University of Missouri Press, Kansas City. Pp. 162-229.

Saenko, S. V., Teyssier, J., van der Marel, D., \& Milinkovitch, M. C. (2013). Precise colocalization of interacting structural and pigmentary elements generates extensive color pattern variation in Phelsuma lizards. BMC Biology, 11, 105.

Sanchez, E., Gippner, S., Vences, M., Preißler, K., Hermanski, I. J., Caspers, B. A., Krause, E. T., Steinfartz, S., \& Kastrup, F.-W. (2018). Automatic quantification of colour proportions in dorsal black-and-yellow coloured amphibians, tested on the fire salamander (Salamandra salamandra). Herpetology Notes, 11, 73-76.

Schneider, M. R., Schmidt-Ullrich, R., \& Paus, R. (2009). The hair follicle as a dynamic miniorgan. Current Biology, 19(3), R132-R142.

Sigmund, W. R. (1983). Female preference for Anolis carolinensis males as a function of dewlap color and background coloration. Journal of Herpetology, 17(2), 137-143.

Stevens, M., Párraga, C. A., Cuthill, I. C., Partridge, J. C., \& Troscianko, T. S. (2007). Using digital photography to study animal coloration. Biological Journal of the Linnean Society, 90(2), 211-237. 
Stevens, M., \& Merilaita, S. (2009). Animal camouflage: Current issues and new perspectives. Philosophical Transactions of the Royal Society B: Biological Sciences, 364(1516), 423-427.

Stevens, M., \& Merilaita, S. (2011). Animal camouflage: Function and mechanisms. In: Stevens, M., \& Merilaita, S. (Eds.). Animal camouflage: Mechanisms and function. Cambridge University Press, New York. Pp. 1-16.

Stuart-Fox, D., \& Moussalli, A. (2009). Camouflage, communication and thermoregulation: Lessons from colour changing organisms. Philosophical Transactions of the Royal Society of London. Series B, Biological Sciences, 364(1516), 463-470

Talbot, J. J. (1977). Habitat selection in two tropical anoline lizards. Herpetologica, $33,114-123$.
Taylor, J. D., \& Hadley, M. E. (1970). Chromatophores and color change in the lizard, Anolis carolinensis. Zeitschrift fur Zellforschung und mikroskopische Anatomie, 104(2), 282-294.

Teyssier, J., Saenko, S. V., van der Marel, D., \& Milinkovitch, M. C. (2015). Photonic crystals cause active colour change in chameleons. Nature Communications, 6,6368

Turing, A. M. (1952). The chemical basis of morphogenesis. Philosophical Transactions of the Royal Society of London. Series B, Biological Sciences, 237(641), 37-72.

Vitt, L. J. (1981). Lizard reproduction: Habitat specificity and constraints on relative clutch mass. The American Naturalist, 117(4), 506-514.

Yang, J., Lu, W., \& Waibel, A. (1997). Skin-color modeling and adaptation. Lecture Notes in Computer Science, 687-694. 